\section{COMMENTS}

The largest review of mediastinal sarcomas consists of 47 patients treated between 1940 and 1991. No mention was made as to how many were pulmonary artery sarcomas. Twelve were malignant peripheral nerve tumors, and only 5 were leiomyosarcomas. Malignant peripheral nerve tumor patients had a $36 \%$ five-year survival rate, whereas none of the other sarcoma patients survived for more than 2 years. ${ }^{1}$

Primary pulmonary artery sarcomas are rare, with fewer than 250 patients described in the literature. ${ }^{2}$ In 1995, the largest series until that time, involving 6 cases, was published from the University of California at San Diego. No patient survived longer than 19 months, even with adjuvant chemotherapy and radiation. The invited commentary for this report suggested that "pulmonary artery sarcomas are almost invariably incurable." 3

More recently, a series and review of the literature from MD Anderson Cancer Center showed encouraging results in 8 pulmonary artery sarcoma patients with bimodality and trimodality therapy and aggressive surgical resection. ${ }^{2}$ All were resected via sternotomy on bypass and all received preoperative or postoperative chemotherapy. Five had replacement of the main pulmonary artery, 3 had pulmonic valve replacement, and 5 underwent pneumonectomy. They reported an estimated median survival of 71 months and an estimated 5-year survival of $72.9 \%$, although no patient was alive and disease free beyond 30 months.

Ninety-two months represents one of the longest disease-free survival intervals in a patient with a pulmonary artery sarcoma, and supports aggressive surgical resection along with multimodality treatment in fit patients. The vast majority of long-term survivors in the literature have been treated with either preoperative or postoperative chemotherapy, and underwent radical resection via median sternotomy on cardiopulmonary bypass, with resection of the main pulmonary artery and/or valve with homograft replacement.

An approach via median sternotomy, with cardiopulmonary bypass available, is necessary to avoid futile thoracotomy in these patients. Although resection of the right or left pulmonary artery can be performed without cardiopulmonary bypass, resection of the main pulmonary artery and even resection and replacement of the pulmonary valve, on cardiopulmonary bypass, should be performed if it is believed that negative margins can be achieved. In this patient, microscopic margins were positive on the left pulmonary artery, but long-term, disease-free survival still was achieved with postoperative radiation.

\section{References}

1. Burt M, Ihde JK, Hajdu SI, Smith JW, Bains MS, Downey R, et al. Primary sarcomas of the mediastinum: results of therapy. J Thorac Cardiovasc Surg. 1998; 115:671-80.

2. Blackmon SH, Rice DC, Correa AM, Mehran R, Putnam JB, Smythe R, et al. Management of primary pulmonary artery sarcomas. Ann Thorac Surg. 2009;87: 977-84.

3. Anderson MB, Kriett JM, Kapelanski DP, Tarzi R, Jamieson SW. Primary pulmonary artery sarcoma: a report of six cases. Ann Thorac Surg. 1995;59: 1487-90.

\title{
Cerebral air embolism during an aircraft flight in a passenger with an air-filled lung cavity associated with remote lung surgery
}

\author{
François-Pierrick Desgranges, MD, ${ }^{\mathrm{a}}$ Martin Cour, MD, ${ }^{\mathrm{a}, \mathrm{b}}$ Romain Hernu, MD, ${ }^{\mathrm{a}}$ \\ Bertrand Delafosse, $\mathrm{MD},{ }^{\mathrm{c}}$ and Laurent Argaud, MD, PhD, ${ }^{\mathrm{a}, \mathrm{b}}$ Lyon, France
}

\footnotetext{
From the Service de Réanimation Médicale, ${ }^{a}$ Hospices Civils de Lyon, Groupement Hospitalier Edouard Herriot, Lyon, France; Faculté de Médecine Lyon-Est, ${ }^{b}$ Université Claude Bernard Lyon I, Lyon, France; and Centre d'Oxygénothérapie Hyperbare, ${ }^{\mathrm{c}}$ Hospices Civils de Lyon, Groupement Hospitalier Edouard Herriot, Lyon, France.

Disclosures: Authors have nothing to disclose with regard to commercial support.

Received for publication March 8, 2013; revisions received April 24, 2013; accepted for publication May 2, 2013; available ahead of print June 24, 2013.

Address for reprints: Laurent Argaud, MD, PhD, Réanimation Médicale, Groupement Hospitalier Edouard Herriot, 5, Place d'Arsonval, 69437 Lyon cedex 03, France (E-mail: laurent.argaud@chu-lyon.fr).

J Thorac Cardiovasc Surg 2013;146:e18-20

$0022-5223 / \$ 36.00$

Copyright (c) 2013 by The American Association for Thoracic Surgery

http://dx.doi.org/10.1016/j.jtcvs.2013.05.008
}

Cerebral air embolism (CAE) is a rare cause of stroke and a potentially life-threatening event. ${ }^{1}$ The incidence of inflight emergencies on commercial airline flights is low. ${ }^{2}$ A few cases of CAE during air travel, associated with the disruption of a bronchogenic cyst, have been reported in the literature. ${ }^{3}$ To our knowledge, we describe the first case of severe CAE during an aircraft flight, secondary to an air pulmonary bulla that developed after lung surgery.

\section{CLINICAL SUMMARY}

A 67-year-old man experienced a sudden loss of consciousness 60 minutes after takeoff of a 2-hour commercial flight. He had a history of lung cancer in the right superior 


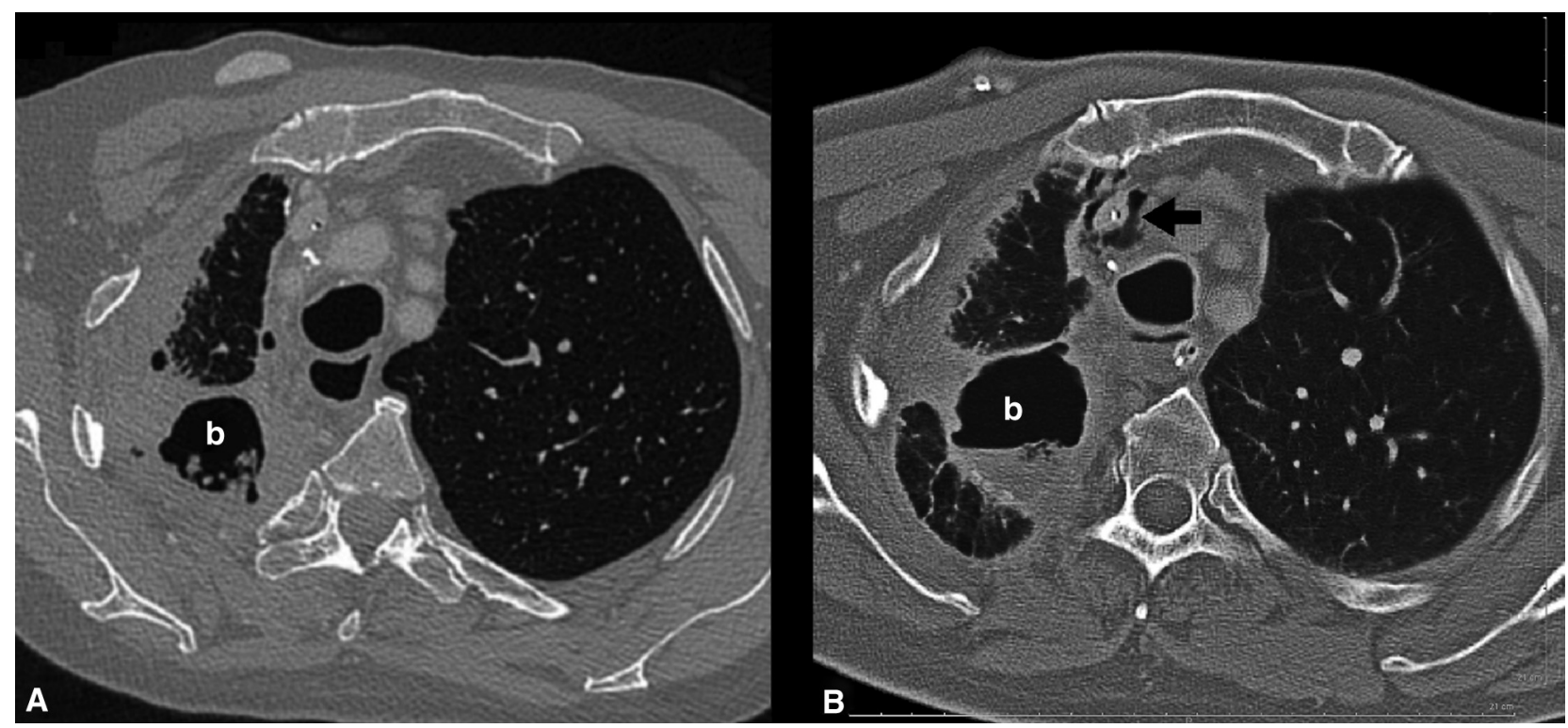

FIGURE 1. Chest CT scan. A, The examination performed 2 months before the flight responsible for the CAE showed the presence of a postoperative asymptomatic bulla $(b)$. B, At admission in the intensive care unit, the examination showed a 6-cm diameter intrapulmonary cavity $(b)$ with an air-fluid level at the apex of the right lung. Laterocervical emphysema surrounding the right jugular vein (black arrow), without evidence of pneumothorax or pneumomediastinum, also is shown.

lobe that was surgically resected 2 years previously. At the airport, he had a Glasgow Coma Score of 4 . The patient was intubated, and mechanical ventilation was started under general anesthesia. On admission to the hospital, a head computed tomography (CT) scan revealed multiple bilateral cerebral hypodensities without brain gas emboli. On further questioning, the patient's wife reported an unexplained episode of syncope during the same flight 1 month before. Because the diagnosis of CAE was suspected, a chest CT scan was performed that showed a 6-cm diameter intrapulmonary cavity in the right lung apex with an air-fluid level and an increase in volume compared with a scan performed 2 months before presentation (Figure 1). The diagnosis of air embolism to the upper body involving the brain, attributable to the seated position during the flight, was accepted. Eight hours after the onset of the neurologic symptoms, a hyperbaric oxygen (HBO) treatment was performed after the insertion of a catheter into the bulla under radiographic guidance to avoid the risk of rupture during recompression. No seizure was observed at any time of the evolution. On day 5 , both head CT scan and brain magnetic resonance imaging confirmed the presence of bilateral ischemic areas consistent with multiple areas of cerebral infarction. Bronchial endoscopy and biopsies showed no evidence of cancer recurrence. Two months later, the patient was discharged with only moderate motor sequelae.

\section{DISCUSSION}

During a flight, and according to the Boyle-Mariotte law (pressure $\times$ volume $=$ constant), the decrease in ambient pressure during the aircraft's ascent generates an expansion of the volume of the enclosed gas. Modern aircraft cabins are pressurized to altitudes up to 8000 feet $(2438 \mathrm{~m})$, increasing the volume of gases in air spaces by approximately $30 \% .^{2}$ If the compliance of the air-filled cavity volume is low, areas of disruption in the peripheral wall may appear, allowing a leakage of air into the surrounding vessels.

In the case reported, the occurrence of an air-fluid level in the bulla with a serohematic fluid in this cavity may evoke a communication with the vascular system. There are 3 broad categories of CAE secondary to the disruption of an air-filled lung cavity that are distinguished by the mechanism of gas entry: (1) from a bronchial vein with occurrence of a paradoxical gas embolism, (2) from a pulmonary vein into the left ventricle, (3) exceptionally from the systemic venous system, that is, the veins surrounding the pulmonary bulla, with a retrograde migration into the jugular veins. ${ }^{1,4}$ Because an echocardiographic study with a bubble test to search for a right-to-left intracardiac shunt was not performed in our patient, the role of a patent foramen ovale cannot be ruled out to explain the exact mechanism of the CAE. Because of the topography of the gas embolism into the cerebral vasculature, it may be simply explained by the position of the head in relation to the torso in a seated position during the flight and the buoyancy of the gas bubbles.

The diagnosis of CAE should be made when there are central neurologic changes and the circumstances are such that a gas embolism could have occurred. When bubbles 
cannot be seen by an imaging technique in the cerebral vessel, the diagnosis remains challenging. In these circumstances, clinical evaluation is still preferred for the diagnosis, and the most important criterion is the patient's history, especially the temporal relation of the onset of symptoms to decompression. ${ }^{1}$ In our case, the acute unexplained neurologic symptoms in the context of exposure to decompression during another flight 1 month before was an additional criterion for the diagnosis of CAE, as well as the clinical presentation and CT scan findings.

Guidelines from the Aerospace Medical Association suggest delaying air travel for 2 to 3 weeks after uncomplicated thoracic surgery. ${ }^{5}$ Moreover, according to the British Thoracic Society, formal medical assessment (including confirmation by chest $\mathrm{x}$-ray of the resolution of any pneumothorax or collected air) is required before departure when a flight is planned by patients who have undergone major thoracic surgery. ${ }^{2}$ In our case, the patient was aware of the presence of a pulmonary bulla (Figure 1). This asymptomatic bulla had been stable in size since lung surgery. No special recommendations regarding air travel were made. An episode of syncope during another flight 1 month before was reported, without a given diagnosis. Thus, patients who are planning to travel by air should be properly advised that a parenchymal bulla is a contraindication of activities that lead to sudden variations of ambient pressure. Our case suggests that the current recommendations might be applied even a long time after a major thoracic surgery in patients with a chronic air collection, especially after previous air travel intolerance.

Despite the lack of prospective randomized studies, recompression treatment with HBO in dedicated centers is the first-line treatment for arterial gas embolism. ${ }^{1}$ Delays in treatment tend to worsen the prognosis, but the effects of delays on the long-term outcome in individual cases are unpredictable. ${ }^{1}$ This therapy decreases the size of intravascular air bubbles by increasing the ambient pressure and allowing nitrogen to diffuse out of the bubble and by causing systemic hyperoxia that allows a better denitrogenation of the cerebral tissues. ${ }^{1}$ Other benefits of HBO have been proposed, such as a better oxygenation of ischemic tissues, a reduction of cerebral edema (by supporting the integrity of the blood-brain barrier), and possibly the inhibition of the adherence of leukocytes to the damaged endothelium. ${ }^{1}$

\section{CONCLUSIONS}

There is a risk of CAE during an aircraft flight in patients with an air-filled lung cavity associated with remote lung surgery. Physicians should be aware of this potential risk of disruption of an air-filled lung cavity secondary to the decrease in ambient pressure and should caution against air travel in patients with pulmonary bullas. An acute stroke in the context of decompression must lead clinicians to evoke the diagnosis of CAE and to search for a history of lung surgery.

\section{References}

1. Muth CM, Shank ES. Gas embolism. N Engl J Med. 2000;342:476-82.

2. Ahmedzai S, Balfour-Lynn IM, Bewick T, Buchdahl R, Coker RK, Cummin AR, et al. British Thoracic Society Standards of Care Committee. Managing passengers with respiratory disease planning air travel: British Thoracic Society recommendations. Thorax. 2011;66:11-30.

3. Closon M, Vivier E, Breynaert C, Duperret S, Branche P, Coulon A, et al. Air embolism during an aircraft flight in a passenger with a pulmonary cyst: a favorable outcome with hyperbaric therapy. Anesthesiology. 2004;101:539-42.

4. Ferry T, Argaud L, Delafosse B, Robert D. Inactive tuberculosis cavity responsible for fatal cerebral air embolism. Intensive Care Med. 2006;32:622-3.

5. Szymanski TJ, Jaklitsch MT, Jacobson F, Mullen GJ, Ferrigno M. Expansion of postoperative pneumothorax and pneumomediastinum: determining when it is safe to fly. Aviat Space Environ Med. 2010;81:423-6. 